\title{
Infektiologie für alle Fälle
}

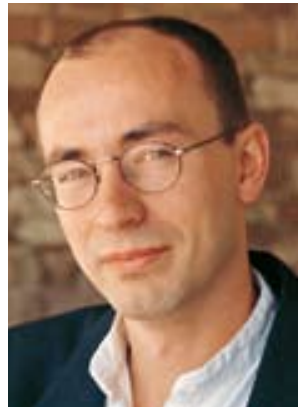

Dr. med. Dieter Schmid, Redaktionsleitung
[1] C.J.L. Murray et al.

Global malaria mortality (...): a systematic analysis.

Lancet 379; 413-431

[2] http://tinyurl.com/79vqzj6
Ich hoffe, Sie haben sich bei der Betrachtung unseres Titelbildes nicht zu sehr erschreckt ... Wobei: Ein gewisser Grusel ist schon angebracht. Die Abbildung zeigt nämlich das Antlitz von Anopheles gambiae, einem der effizientesten Überträger der Malaria. Wie keine andere Anopheles-Art sticht sie - angelockt vom typischen Käsegeruch ungewaschener Zweibeinerfüße - mit Vorliebe Menschen. Laut aktuellen Berechnungen von US-Forschern verursachen die Plasmodien, die durch die Stechrüssel solcher Mücken in den Körper von Menschen injiziert werden, pro Jahr 1,24 Millionen Todesfälle [1] - fast doppelt so viele, wie die WHO bislang vermutet hat. Diese Hiobsbotschaft sollte ein starker Impuls sein, sofort den Kampf gegen diese Krankheit zu intensivieren. Doch Fehlanzeige: Forscher, die nach neuen Therapien gegen Malaria oder ähnlichen Geißeln aus ärmeren Teilen der Welt suchen, haben derzeit wegen der Finanzkrise sogar weniger Gelder zur Verfügung. Das spürt auch der „Globale Fonds“, das wichtigste internationale Programm zur Bekämpfung der schweren Infektionskrankheiten Malaria, AIDS und Tuberkulose. Er tut sich immer schwerer, Mittel für neue Förderprogramme aufzutreiben [2].

Einen Weg, wie Sie ganz persönlich gegen diesen Missstand ankämpfen können, beschreiben wir in unserem Artikel „Heilkunst für die heißen Breiten“ (S. 24). Als Tropenmediziner können Sie aktiv an der Entwicklung neuer Konzepte arbeiten, die die Plasmodien, Trypanosomen und Ankylostomen dieser Welt zurückdrängen. Und nicht nur das! Seit einigen Jahren suchen Tropenärzte auch nach Lösungen für die wachsenden nichtinfektiösen Gesundheitsprobleme der Tropen - zum Beispiel Adipositas oder Diabetes mellitus Typ II aufgrund kalorienreicher Fehlernährung. Die prinzipiellen Methoden, die man bei solchen sozialmedizinischen Fragestellungen anwendet, sind von denen der Infektiologie eigentlich gar nicht so weit entfernt: Auch hier geht es darum, „Krankmacher“, die eine Gesellschaft befallen haben, aufzuspüren - und zu beseitigen. In diesem Heft berichtet eine Studentin zum Beispiel von ihrer Promotion über die gesundheitliche Situation der Frauen in Nicaragua (S. 12). Diese ist miserabel, ausgelöst durch eine „Mischinfektion“ aus religiöser Verblendung, mangelnder Bildung und einer tief verankerten Macho-Kultur.

Was lernen wir daraus? Der Stich von Anopheles gambiae gehört sicher zu den größten Gesundheitsrisiken. Ich denke aber, mindestens genauso gefährlich ist die weltweit grassierende „Pandemie“ aus fehlendem Gesundheitswissen. Wer dagegen ankämpfen möchte, muss kein neues Antibiotikum erfinden - es reicht schon, Menschen zu erklären, wie sie gesund bleiben können!

Ein gutes Sommersemester wünscht

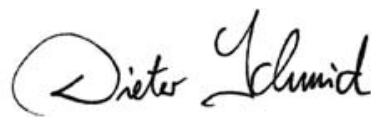

ONLINE-UMFRAGE

\section{PJ: Allgemeinmedizin als Pflicht-Tertial?}

Das Praktische Jahr ist der Höhepunkt des Studiums: Endlich darf man selbst Hand am Patienten anlegen und sich - in engen Grenzen als aktiver Mediziner versuchen. Bislang durchlaufen die PJler jeweils16 Wochen die Chirurgie und die Innere Medizin. Das Fach fürs dritte Tertial ist frei wählbar. Genau dies soll sich laut den Landesgesundheitsministerien nun aber ändern: Um mehr Jungärzte für die hausärztliche Medizin zu begeistern, wollen sie ein verpflichtendes Tertial in einer Allgemeinarztpraxis vorschreiben.
Verbände wie die Bundesvertretung der Medizinstudierenden in Deutschland (bvmd) laufen dagegen Sturm: Zum einen seien die Allgemeinpraxen gar nicht auf die Lehre ausgerichtet und zum anderen führe das Pflichttertial zu Engpässen in anderen Fächern. Was ist Ihre Meinung?

- Sollte das dritte Tertial verpflichtend in einer allgemeinmedizinischen Praxis stattfinden?

Stellen Sie sich dieser Frage und kommentieren Sie unter: www.thieme.de/viamedici/aktuelles/ aktion/miniumfrage2-12.html
- Ergebnis der Umfrage in Via medici 1.12: Wir fragten, was Sie sich für Ihre erste Stelle als Assistenzarzt wünschen würden, wenn Sie bei einer guten Medizinerfee drei Wünsche frei hätten. Ganz vorne lag die Sehnsucht nach einem guten kollegialen Verhältnis. 25\% der Teilnehmer äußerten diesen Wunsch. Knapp dahinter rangierte mit 22\% die gute Betreuung während des Einstiegs. Für $14 \%$ ist eine strukturierte Weiterbildung sehr wichtig. Kein einziger Teilnehmer wünschte sich, in einer Klinik auf dem flachen Land anzufangen. Mehr zur Umfrage unter:

.../aktuelles/aktion/miniumfrage1-12.html 